Geopolítica(s) Revista de estudios sobre espacio y poder ISSN: 2172-3958

https://dx.doi.org/10.5209/geop.72011

\title{
El testimonio de un intento inteligente, paciente y humano por fundar una paz positiva en Colombia
}

Miguel Barreto Henríquez (2016) Laboratorios de paz en territorios de violencia(s). Bogotá: Universidad de Bogotá Jorge Tadeo Lozano, 548 pp. ISBN: 978-958-725-192-0.

Este extraordinario trabajo de Miguel Barreto Henríquez es un testimonio del intento concreto, inteligente, paciente y humano, de unas personas de un par de regiones humildes y conflictuadas de Colombia, por fundar una paz positiva, por medios alternativos a los planteados por el gobierno de Álvaro Uribe Vélez en el período 2002-2010.

En efecto, el trabajo narra la experiencia inédita de construcción de paz llevada a efecto por los llamados Laboratorios de Paz que, íntimamente conectados con la experiencia previa de los Programas de Desarrollo y Paz colombianos, fueron concebidos como sistemas multidimensionales de búsqueda de paz desde la base, básicamente sostenidos por la sociedad civil, aunque respaldados por la Unión Europea y la República de Colombia.

Esta vinculación de la génesis de los laboratorios de paz con las políticas de cooperación al desarrollo y política exterior de la Unión Europea, unida a la pregunta central del texto acerca de la eficacia de los laboratorios para abrir un camino alternativo a la paz, permiten inscribir al trabajo de Barreto Henríquez dentro del debate acerca de si, para ser más efectiva, la democracy assistance debe ser de carácter más político o normativo, más desarrollista o inclinada hacia preocupaciones socio económicas o, más francamente, un complemento dialógico de ambos enfoques ${ }^{1}$. Una discusión siempre actual y pertinente en la región latinoamericana.

Como la idea que llena de ritmo y significado al texto es la paz como fruto de la inclusión democrática, su marco teórico es un juego a contrapunto entre la corriente teórica más popular de gestión de conflictos, por un lado; y los enfoques alternativos de resolución y transformación de estos últimos derivados directamente de la peace research, por otro, en los que transformar indica un proceso sistemático de inclusión con impacto en cuatro dimensiones: la personal, relacional, estructural y cultural.

El resultado de este contrapunto es uno de los capítulos más hermosos del libro que, a través de un ejercicio puramente descriptivo, consigue transmitir las claves del carácter incompleto del paradigma dominante de la gestión de conflictos para

1 T. Carothers: Democracy Assistance: Political vs. Developmental? Journal of Democracy, 20(1), 2009, 5-19. 
alcanzar la paz duradera, revelando a su vez la complementariedad y sensibilidad necesarias de los enfoques estructurales e inclusivos para la construcción de la paz. Según Barreto Henríquez, el primer paradigma, realista y Estado-céntrico, cuenta escasamente con las herramientas de la negociación o bargaining y la coacción y, bajo el supuesto de atribución de la culpa de los conflictos a colectivos y personas - no a sistemas institucionales ni políticas-, plantea un marco de seguridad y criminalidad a la salida de los conflictos.

Resolver conflictos, en cambio, significa desanudar incompatibilidades, buscar causas y soluciones y atender los problemas subyacentes. Y para John Burton, Johan Galtung y John Paul Lederach, los idealistas de la paz, transformarlos es satisfacer necesidades e intereses explícitos, procurar el crecimiento de la justicia social y aumentar las capacidades de realización somática y mental del individuo. ¿Por qué? Porque para ellos la paz es autorrealización o emancipación social y la violencia no es solo aquella manifestada externa y directamente, sino toda realidad en la que hay sufrimiento y exclusión, aunque este sufrimiento no tenga autor o sea simplemente imputable a la forma que hemos llegado a adoptar como sociedad. Lo que no significa que se pueda vivir sin conflicto. Sin conflicto no hay democracia, y todo intento de imponer una visión única de la sociedad implica lo contrario. En este contexto, sin embargo, la exclusión de la violencia como método de zanjar diferencias, destaca como el único de los prerrequisitos consensuados necesario para alcanzar una sociedad democrática e inclusiva, ya que nadie debería poder imponerse por la violencia ${ }^{2}$.

Los enfoques históricamente aplicados en los intentos de resolución del conflicto colombiano los resume el capítulo segundo. El autor los reduce a dos. En el enfoque militar, la insurgencia es tratada como un problema de estricto orden público y no de orden social. Las últimas aplicaciones de este enfoque fueron el Plan Colombia y la Política de Defensa y Seguridad Democrática, muy exitosas en lo militar, pero reincidentes en el patrón de exclusión de la transformación social de la comunidad política como uno de sus fines. Por su parte, se ejecutó también un enfoque de negociación del que se emplearon dos subtipos. El primer subtipo fue el esquema de negociación con agenda limitada, centrado en la inclusión política de la insurgencia, con exclusión de métodos de inclusión económica y social. El segundo, fue el esquema de negociación con agenda amplia que, no obstante su nombre, siguió extrañando dos cosas. La primera, la incorporación de actores de base cuya consideración resulta esencial para entender la complejidad del conflicto; la segunda, la mayor de las voluntades políticas del Estado para alcanzar transformaciones estructurales.

La clave de la armazón del trabajo es la relación de dependencia que el autor establece, como es natural, entre el tipo de lectura que se tenga del conflicto y el resultado del examen de la efectividad de los laboratorios de paz. Dicho de otra forma, el éxito en la proposición de las políticas de paz tiene su centro en la reducción del error en la interpretación del conflicto armado. Como los Programas de Desarrollo y Paz que, como observa Barreto Henríquez, en el fondo fueron en gran medida auspiciados por la UE y, por tanto, de marcado acento desarrollista, se desprende de allí un concurso en el diagnóstico del origen de la violencia en Colombia 
que tanto el autor como el programa comparten. Este origen estaría en causas estructurales de exclusión económica, política, social y regional, de las que parten para ofrecer soluciones. De esta coincidencia se desprende también el gran entusiasmo que el autor del texto transmite.

En este sentido, en el capítulo tercero se abre un debate antideterminista sobre las causas profundas del conflicto colombiano, del que surge un consenso con amplios sectores de la doctrina internacional según el cual, cuando se restablecieron las democracias de Venezuela y Colombia en medio de circunstancias adversas, no obstante las constituciones escritas establecían gobiernos presidenciales, los líderes de los principales partidos recurrieron rápidamente a acuerdos de asociación para suavizar las duras implicaciones del juego electoral suma-cero. Como muestra especialmente el caso colombiano, al hacerlo, se mantuvieron excluidas durante mucho tiempo a ciertas minorías, privándolas de su libertad de elección ${ }^{3}$. Venezuela, gracias al petróleo, pudo de forma temporal resolver sus problemas institucionalmente. Colombia, sin embargo, pasaría más rápidamente a la resolución violenta de sus conflictos y exclusiones, en un contexto en que la impunidad continuada deslegitimaba reiteradamente al Estado.

Mención especial merece otro de los consensos doctrinarios en torno al elemento regional como factor de exclusión del desarrollo. Son varias las razones por las que, en este contexto, el Estado colombiano ha estado presente diferencialmente en la geografía colombiana. Además del factor geográfico per se, la fuerza acumulada de diversos factores locales ha terminado por discutir la unidad del Estado-Nación en Colombia. Asimismo, otra de las claves del conflicto nacional obedece a los órdenes sociales locales, creados en ausencia del Estado central.

En el fondo, tanto en la lectura de las causas profundas del conflicto colombiano, como en la longevidad del conflicto y sus actores, incluida la edad de las guerrillas, subyace otro gran punto de coincidencia con cierta doctrina sobre un hecho más amplio: cambiar las características del Estado es mucho más difícil que cambiar de régimen político. La legitimidad de muchas democracias del mundo pareciera depender menos de la profundización de sus instituciones democráticas, que de su capacidad de proporcionar una gobernanza de alta calidad, por lo que la clave del bajo rendimiento de ciertos regímenes democráticos latinoamericanos podría encontrarse en haber fallado en el establecimiento de Estados modernos e inclusivos y la incapacidad de ofrecer bienes públicos básicos como educación, infraestructuras y seguridad ciudadana ${ }^{4}$.

El cuarto capítulo explica la forma de concebir los laboratorios de paz desde la perspectiva europea, en el contexto amplio de las políticas de paz comunitarias hacia Colombia. Aquí se reflejan las principales fortalezas potenciales del enfoque desarrollista esencialmente europeo. La primera, un énfasis en el cambio gradual a pequeña escala y en sectores menos politizados que puede permitir la entrada de los proveedores de asistencia internacional en situaciones políticas tensas y restrictivas, donde un enfoque preponderantemente político podría provocar menor receptividad. En segundo lugar, una orientación de largo plazo intrínseca al tipo de compromiso sostenido que el enfoque de desarrollo fomenta y que es necesario

J. J. Linz: The Perils of Presidentialism. Journal of Democracy, 1(1), 1990, 51-69.

F. Fukuyama: Why Is Democracy Performing So Poorly? Journal of Democracy, 26(1), 2015, 11-20. 
para el cambio en muchos sectores como podría ser, por ejemplo, el autodescubrirse como ciudadano, donde los esfuerzos a corto plazo rara vez producen resultados - esto último hecho posible gracias al contexto de dinámicas de reforma positiva ofrecido por los laboratorios de paz-. En tercer lugar, la adopción de una visión amplia de la democracia que predispone hacia el fomento e identificación de conexiones útiles entre reforma política y reforma socioeconómica ${ }^{5}$. Por último, una inclinación hacia la no militarización de los problemas socioeconómicos que pone de relieve la importancia de la participación activa de la sociedad civil, en este caso, en el proceso de pacificación.

Finalmente, en los capítulos quinto y sexto se describen las experiencias vividas por el autor dentro de los laboratorios. El autor advierte que la metodología que condujo su trabajo fue eminentemente empírica, con base en entrevistas a participantes y actores de aquéllos, así como observación participante en eventos e iniciativas propias de sus dos estudios de caso. El primer laboratorio, el del Magdalena Medio, se constituyó en una sola región diversa, campesina y, por tanto, geoestratégicamente clave para el conflicto armado. Barrancabermeja, su ciudad más grande, se distingue por ser la capital petrolera y obrera del país porque $70 \%$ de los crudos de Colombia se refinan allí. Por tanto, la primera marca que este laboratorio llevó fue el hiriente contraste entre la mucha concentración de capital y la mucha pobreza, con la única presencia estatal del ejército cuidando refinerías y persiguiendo campesinos, dentro de un esquema económico extractivo y de enclave.

El segundo laboratorio fue el del Cauca y Nariño (Macizo Colombiano y Alto Patía) que, a diferencia del primero, no se estableció en una, sino en tres regiones del país, lo que haría evidente desde su constitución no solo la diversidad de intereses de cada entidad territorial participante y la unión artificial de las entidades creadas ad hoc con órganos de una y otra, sino la ausencia de una conformidad indígena compacta, dividida por los niveles de confiabilidad que cada cual otorgaba al gobierno central como actor de la iniciativa.

Ambos laboratorios estuvieron unidos por los propósitos de contribuir con la convivencia ciudadana y mostrar trayectos eficaces y viables en el camino hacia la paz, mediante la defensa de los derechos humanos de todos los habitantes del lugar y el estímulo al desarrollo humano sostenible. También compartieron filosofía; una en la que, por un lado, la reconstrucción del Estado-Nación colombiano es tan posible como posible es acercarlo a las regiones donde los excluidos padecen y las desigualdades abundan. Por otro, una en la que la paz existe siempre que esté en unión con el desarrollo. De hecho, la receta para la paz es el desarrollo incluyente y sostenible, solo que la relación entre ambas variables está mediada por la desigualdad, la acumulación del poder político, la calidad institucional y la cantidad y nivel de prestación de los servicios públicos. Por último, los laboratorios compartieron también un tipo de metodología incluyente porque partieron de la idea de que la paz es un bien colectivo y la participación ciudadana, parte del proceso de desarro1lo. En el caso del primer laboratorio, mucho más que en el segundo, el autor afirma haber encontrado la influencia filosófico-religiosa de Francisco de Roux, de quien podría afirmarse que lucha más en términos de consolidar una ética pública que una de tipo religioso, y a quien el autor describe como perteneciente a un sec- 
tor progresista y radicalizado de la Iglesia católica movilizada por la opción por los pobres; una especie de socialismo cristiano.

Es imposible comprimir aquí la cantidad de proyectos y procesos alumbrados por los laboratorios, aunque sí que es factible figurarse su envergadura si se presta atención a las diferentes dimensiones que agruparon sus componentes. En primer lugar, abordaron la dimensión política de la construcción de la paz mediante el diseño y creación de escenarios de paz, concertación y derechos humanos. Estos, esencialmente, fueron espacios socio-territoriales que buscaron la cohesión interna de la comunidad frente al conflicto, la identificación de las causas estructurales del conflicto por parte de la comunidad y el desarrollo de la conciencia de que existían medios sociales y culturales para enfrentarlas. En segundo lugar, trataron la dimensión social, cultural e institucional de la construcción de paz que, además de radios comunitarias, puso en marcha un inventario de recursos dirigidos a la mente y percepción de los vecinos, además de otros procesos sociales y de gobernabilidad democrática. Por último, afrontaron la dimensión socio-económica, ayudando a emprender proyectos productivos y ambientales para el desarrollo sostenible.

Cada laboratorio tuvo una cantidad de logros cuantificables muy estimulantes, entre ellos: 150.000 beneficiarios, 1.700 puestos de trabajo directos y 230 indirectos, el aumento del ingreso de los participantes en un $50 \%, 4.300$ líderes formados, 100 organizaciones comunitarias participantes, 200 alianzas, 2.500 familias insertas en proyectos productivos, 11.578 hectáreas cultivadas, 9.170 obras de infraestructura, 12 unidades de salud reestructuradas. Pero ninguno de los anteriores se puede conjugar como se hace con ciertos logros cualitativos: los laboratorios ofrecieron alternativas al conflicto, deslegitimaron la violencia, legitimaron la paz, robaron hombres y espacio a la guerra, acercaron instituciones a los ciudadanos, integraron a la comunidad y dieron cabida a la comprensión de la existencia de un otro a nivel cultural, simbólico, lingüístico y afectivo, fundamental para que las diferencias interactúen y trasciendan a un algo mejor.

Hablando de alteridades, aparte del elemento geográfico mencionado más arriba, la composición etnográfica fue uno de los elementos que diferenció al segundo laboratorio. Este último también se distinguiría por tener un mayor grado de centralización y verticalidad y, proporcionalmente, un menor grado de autonomía que el primero; dependencia que se materializó en el manejo de los recursos burocráticos y financieros y en el esfuerzo del gobierno del expresidente Álvaro Uribe por sacar los temas políticos del alcance del laboratorio, por reducirlo a la consideración exclusiva de temas sociales y, en ese sentido, convertirlo en un mero apéndice del programa de recuperación de territorios que por entonces ejecutaba el gobierno central.

Al texto de Barreto Henríquez lo surcan transversalmente dos hermosísimas ideas, imprescindibles para entenderlo y comprender el alcance de los laboratorios. Sobre la noción de desarrollo dice, citando a Francisco de Roux, que éste es "la expansión sostenible de las posibilidades de la dignidad humana en un territorio" (p.283). El otro surco lo traza la idea de que, como personas, nos desplazamos no solo física sino moralmente. Por tanto, la razón última de cada laboratorio era evitar los desplazamientos; evitar que la gente se ausentase del lugar material y espiritualmente; ganarle espacios a la guerra dentro y fuera del individuo. Ya hemos dicho que el libro insiste en que a los conflictos solo es posible superarlos trans- 
formándolos. He aquí donde se cierra el círculo del razonamiento del autor: transformando las estructuras de exclusión, se hace posible elevar la dignidad de las personas.

En el capítulo séptimo, el autor orienta un diálogo entre los niveles macro del conflicto armado colombiano y el nivel micro de los laboratorios. Su clave de lectura está en la asimilación del hecho de que, en cuanto se traten como categorías separadas, las estrategias centrales y locales tendrán siempre limitaciones. Se pierde mucha capacidad de análisis del poder alto y el poder de base si cada uno se aísla. Los laboratorios constituyeron experiencias de descentralización de la transformación de un conflicto que, aunque aspiró siempre a alcanzar el horizonte de orquestación nacional, dependió mucho de su capacidad para contagiar entusiasmo a tales niveles. Quizá por ello el autor señale como principales limitaciones de su objeto de estudio, el ámbito geográfico de intervención micro del Programa de Desarrollo y Paz que, por su carácter restringido, se vio impedido de transformar e influir en causas de exclusión nacionales y estaduales.

Pero existen causas de exclusión macro que, una vez descompuestas en sus elementos estructurales, arrojan dimensiones geográficas de acción local por excelencia. La igualdad de las personas ante la ley, derivada a su vez de la idea de Estado de Derecho, es una de ellas ${ }^{6}$. Una vez descompuesta en partes, la noción de igualdad permanece vinculada con los derechos humanos y civiles, el aspecto social, y el desempeño general de las instituciones oficiales, y no hay duda de que cada uno de estos ámbitos reclama un contexto de actuación micro, concreto, que tiene a todo ciudadano individualmente considerado y a toda comunidad como beneficiario y protagonista. ¿No apuntó hacia ellos el esfuerzo de cada laboratorio de paz en cada una de las regiones intervenidas? Definitivamente. Por lo que el libro nos deja en cierto sentido una visión más optimista que la del propio Barreto Henríquez en cuanto al impacto del modelo y nos reafirma la adecuación de los enfoques de las soluciones, surgidas del diagnóstico del conflicto.

Quizá lo único que se echa de menos en el libro haya sido un análisis breve de cómo la arquitectura constitucional colombiana haya podido contribuir o dificultar el esfuerzo descentralizador de los laboratorios. A fin de cuentas, todo esfuerzo por conseguir la paz exige también un soporte institucional lo suficientemente complejo, coherente y flexible, como para llenar a la gente de toda la autonomía necesaria para sentir que el poder también es suyo y que, como ciudadano, ha sido tomado en cuenta.

Quedan en el aire las preguntas de si los laboratorios conservan su sentido luego de haber sido alcanzados los acuerdos de paz de 2016 y si son útiles al postconflicto y en qué medida. Son preguntas lógicas, aunque carentes de sentido de la realidad, a las que conviene responder con otras preguntas. ¿Acaso se han extinguido las violencias en Colombia? ¿Qué dicen de ellas lo que va de postconflicto, el descubrimiento de los abusos de militares a niñas indígenas, la presencia actual de guerrillas, disidencias y autodefensas, el asesinato continuado de líderes sociales, las recién descubiertas redes de espionaje contra periodistas, las ejecuciones extrajudiciales y la espeluznante circular oficial que instaba a los militares a mostrar

G. O'Donnell: The Quality of Democracy: Why the Rule of Law Matters. Journal of Democracy, 15(4), 2004, $32-46$. 
resultados? ¿Por qué los uniformados siguen siendo los únicos en llegar a las regiones y no el Estado? ¿Ha llegado la paz positiva a aquéllas? Mientras las causas estructurales del conflicto sigan allí, el trabajo de Barreto Henríquez y el de los laboratorios, no solo permanecerán vigentes, sino que seguirán siendo el punto de partida recurrente, la guía práctica surgida de un gran esfuerzo creativo.

Héctor José Pantoja Pérez-Limardo Facultad de Ciencias Políticas y Sociología Universidad Complutense de Madrid Email: hectorjp@ucm.es 